\title{
A VULNERABILIDADE DOS IDOSOS ÀS DOENÇAS PSICOSSOMÁTICAS E À PRECOCIDADE SENIL
}

\section{ARTIGO ORIGINAL}

SANTOS, Francisco Medrado ${ }^{1}$

SANTOS, Francisco Medrado. A vulnerabilidade dos idosos às doenças psicossomáticas e à precocidade senil. Revista Científica Multidisciplinar Núcleo do Conhecimento. Ano 03, Ed. 09, Vol. 11, pp. 05-14 Setembro de 2018. ISSN:24480959, Link de acesso: https://www.nucleodoconhecimento.com.br/saude/doencaspsicossomaticas, DOI: 10.32749/nucleodoconhecimento.com.br/saude/doencaspsicossomaticas

\section{RESUMO}

O envelhecimento da população tem sido uma questão importante à ser discutida na área da saúde, exigindo cada dia mais preparo dos profissionais, em como diagnosticar as patologias e no cuidar, pois com o envelhecer, o indivíduo apresenta deficiências mentais e orgânicas corporal tornando-os vulneráveis às doenças psicossomáticas e em consequências disto a senilidade que pode vir de forma precocemente, sendo resultado de situações de ordem ambiental e social vividas, ao longo do tempo, tendo como consequências os agravos à saúde. Este estudo teve como objetivo conhecer quais as vulnerabilidades do idoso às doenças psicossomáticas e como elas podem contribuir para a senilidade do idoso, tendo como justificativa a importância de conhecer estes agravos à saúde, para precocemente ajudar esta população em suas deficiências, sabendo que nem todo idoso é senil. É um estudo de característica bibliográfica e qualitativa, por exploração de artigos científicos divulgados nos últimos 5 anos, no Brasil, sendo encontrados nas seguintes bibliotecas online: LILACS, PEPSIC, SBGG, SCIELO, UFRGS, UNICAMP, E USP|SP. Tendo como descritores: idoso, doenças psicossomáticas,

\footnotetext{
${ }^{1}$ Enfermagem/docente
}

RC: 21029

Disponível em: https://www.nucleodoconhecimento.com.br/saude/doencaspsicossomaticas 
senilidade e vulnerabilidade. Este estudo mostrou que os fatores ambientais, e sócio econômico contribuem para a vulnerabilidade do idoso, às doenças psicossomáticas e a contribuição destas para a senilidade.

Palavras chave: doenças psicossomáticas, Idoso, Senilidade, Vulnerabilidade.

\section{INTRODUÇÃO}

A população idosa tem se multiplicado significativamente não só no Brasil, mas também em todo o mundo, conforme citado por (GONÇALVES, 2015, p. 645- 646), este acontecimento tem sido uma conquista da humanidade, graças aos avanços tecnológicos, que tem possibilitado ofertar melhores condições de vida, voltados à atenção da saúde, não resultando apenas do aumento da longevidade, mas também do significante declínio da fecundidade. Isto sugere que há a necessidade de se procurar com mais afinidade o conhecimento sobre esta população, seus anseios, suas deficiências, e as vulnerabilidades aos agravos à sua saúde, que podem ser entre vários fatores, os psicológicos, somatizando em seu corpo a senilidade, ou seja, os processos patológicos.

Este estudo teve como objetivo conhecer quais as vulnerabilidades do idoso às doenças psicossomáticas e como elas podem contribuir para o processo da senilidade. Pois são consequências de fatores de ordem ambiental, social, econômico e cultural, que são acumulados no histórico de vida do indivíduo, que ao atingir a idade madura sofreu um significativo desgaste não só no corpo, mas também no emocional, agora começa a vir à tona os seus resultados em forma de agravos à saúde. O que justifica este estudo é que, tendo em vista o aumento significativo da população idosa no Brasil, estamos diante de uma grande problemática a ser encarada quanto as assistências à saúde e também social, deste grande contingente de pessoas, havendo a necessidade de capacitação de profissionais da saúde para diagnosticar, e tratar o mais precocemente possível e com maior competência, não só os problemas crônico-degenerativos que esta população tem apresentado frequentemente, mas também os de origem 
psicossomáticas que também tem a sua participação neste processo de vulnerabilidade e senilidade. O processo natural do envelhecimento humano, o qual é conhecido como Senescência ou Senectude, ocorre desde o nascimento, e isto acontece gradualmente levando o corpo e a mente a "sofrer" mudanças que são inevitáveis, contribuindo para o seu amadurecimento e até mesmo para a sua saúde. Este indivíduo vai ser moldado para uma vida saudável pelas suas interações com o ambiente em que convive ao longo de sua vida, sendo importante também as suas interações sociais e culturais vividas na formação de sua história. Tudo isto vai num futuro bem próximo apresentar um resultado positivo ou negativo, quanto ao estado de saúde. Ao chegar à idade adulta, o corpo humano começa à apresentar declínios gradativamente nas suas estruturas e no funcionamento das suas funções orgânicas, onde podem acontecer diminuição visual, olfativa, auditiva, gustativa, tegumentar, cognitiva, etc., e consequentemente as vulnerabilidades aos agravos à saúde, pelas deficiências funcionais do organismo, isto não quer dizer que o idoso é senil, sendo apenas vulnerável à este processo. (CLOSAK ET ALL, 2011, p. 1765), argumenta que não é correta a associação da velhice com os processos patológicos, "pois mesmo existindo perdas, tanto no nível biológico, como econômico, social e psicológico, a manutenção das atividades e do engajamento social e familiar favorece o envelhecimento saudável". Não há interligação entre a Senescência e a Senilidade como muitos erroneamente pensam, pensando que todo idoso é senil, entendendo que as demências, as incontinências urinárias e fecais, e outros agravos à saúde do idoso sejam pelo fato deste ter envelhecido. A Senilidade é um outro processo do envelhecimento, chamado de processo patológico pelas alterações decorrentes de doenças mentais ou crônicas como Alzheimer, Depressão, hipertensão arterial, diabetes, câncer, alterações tegumentares, etc.

Sendo importante saber que, a grande diferença entre a Senescência e a Senilidade é que, no primeiro processo o idoso apresenta gradativamente perdas de vitalidade dos órgãos e sistemas, sem o acometimento de qualquer doença, enquanto que no segundo processo as doenças acometem o idoso em consequência destas perdas e associado a diversos fatores. A vulnerabilidade é a predisposição que este indivíduo 
no processo de Senectude apresenta nesta faixa etária de apresentar os agravos à saúde, passando então para o processo de Senilidade. Isto reforça o pensamento de que nem todo idoso é senil, mas que todo idoso é vulnerável a senilidade mental ou corporal, pelas perdas da vitalidade funcional da mente e do corpo.

\section{DESENVOLVIMENTO}

Este estudo trate-se de uma pesquisa bibliográfica, qualitativa, com a exploração de artigos científicos publicados nos últimos 5 anos, no Brasil. Para tal estudo houve a seleção de 12 artigos. Tais fontes de informações foram encontradas em bancos de dados online, a saber: LILACS, PEPSIC, SBGG, SCIELO, UFRGS, UNICAMP, E USP/SP, sendo destacados como descritores: doenças psicossomáticas. Idosos. Senilidade. Vulnerabilidade. Nos últimos 50 anos, o número da população idosa no Brasil tem aumentado significativamente em comparação ao número de crianças e adolescentes, o envelhecimento desta população são atribuídos à grande queda da fecundidade que ocorreu de 1960 à 2012, e também pela redução significativa dos casos de mortalidade infantil ocorridos neste mesmo período (DOLL, RAMOS \& BUAES, 2015, P. 9); Os autores ainda enfatizam que, estes fatores tiveram como contribuintes as mudanças no campo social, na educação, na cultura e na saúde, tendo como exemplo a descoberta do antibiótico, as vacinas, a formação de unidades de terapias intensivas e ainda, mudanças de estilos de vida das pessoas. (ALONSO, 2011, P. 1), o envelhecimento da população é um fenômeno de ordem mundial, o idoso tem um papel importante de relacionamento na social, e na esfera familiar, eis aí um importante valioso objeto de estudo, principalmente na esfera acadêmica tornando-se um campo primordial. Neste caso, a Demografia se apresenta como um campo importante de estudo para tal temática, este fenômeno é de extrema relevância pois produz importante impacto na população, como nova modelagem de sua estrutura etária, novas demandas no planejamento urbano, dentre outros fatores. Conforme citado por (CLOSAK ET ALL, 2011, p. 1764), o significativo aumento da população idosa também está relacionado ao avanço da tecnologia, e aos tratamentos de doenças cronicas como as Doenças e Agravos Não 
Transmissíveis (DANT), resultando em um aumento do contingente de indivíduos com mais de 60 anos. Conforme descrevem os autores, o envelhecimento é um processo natural do indivíduo implicando em mudanças graduais inevitáveis no corpo do indivíduo devido à sua idade, provocando desgastes no seu organismo, causando the várias alterações nas esferas culturais, sociais e emocionais. Segundo (GONÇALVES, 2015, p. 645-647), é um processo de ordem universal em que todos os indivíduos passam, de forma irreversível, sendo gradualmente "sofrido", de forma complexa, sendo afetados nas dimensões fisiológicas, psicológicas e também sociais. Quando o envelhecimento é encarado como um processo contínuo, temos a consciente realidade de que a cada hora, e também todos os dias envelhecemos, sem termos necessariamente a ideia de sermos velhos.

Para (CLOSAK ET ALL, 2011, p. 1764), apud (Organização Mundial da Saúde 2006) o envelhecimento é:

Um processo sequencial, individual, cumulativo, irreversível, universal, não patológico de deterioração de um organismo maduro, próprio a todos os membros de uma espécie, de maneira que o tempo o torne menos capaz de fazer frente ao estresse do meio ambiente.

(BARBOSA, 2012, P. 49), Refere que houve no Brasil um acelerado envelhecimento da população, tendo como fator responsável a equação da queda significativa da fecundidade mais a soma expressiva dos indivíduos com sobrevida maiores de 65 anos, salientando que o país está diante de uma grande problemática a ser encarada quanto as assistências à saúde e também social, devido a este grande contingente de pessoas nesta idade, havendo a necessidade de capacitação profissionais da saúde para diagnosticar, e tratar o mais precocemente possível e com maior competência, os problemas crônico degenerativos que esta população tem apresentado frequentemente.

Diante desta realidade, é imprescindível que haja políticas públicas voltadas para atender às necessidades humanas básicas desta população, e que se conheça as vulnerabilidades desta população aos agravos à sua saúde e as possíveis consequências destes agravos, na intenção de promover qualidade de vida.

RC: 21029

Disponível em: https://www.nucleodoconhecimento.com.br/saude/doencaspsicossomaticas 


\section{A PSICOSSOMÁTICA E AS VULNERABILIDADES}

(SANTOS, 2012, P. 7), Define a psicossomática como sendo uma prática integral da medicina que usa como campo de pesquisa o corpo e a mente na compreensão da interação destes no processo do adoecimento. O próprio envelhecimento do indivíduo é um fator de risco para que nele se desenvolva vulnerabilidades de ordem biológica, socioeconômica, cultural ou mesmo psicossocial, como consequência do declínio biológico natural, típico deste processo, somatizando a interação de processos socioculturais, sofrendo efeitos acumulativos de deficit educacional, de poder aquisitivo e de problemas de saúde não resolvidos ao longo de sua vida e até mesmo devido aos estilos de vida atual. Tanto os aspectos individuais, quanto coletivos, contextuais ou mesmo os históricos de vida de desenvolvimento e de envelhecimento que este indivíduo tem vivenciado, podem ser fatores de risco do seu adoecimento. (SCHUMACHER, PUTTINI \& NOJIMOTO, 2013, P. 282), apud (TOWNSEND, 1980), o fato de impor a aceitação da aposentadoria precoce, tirar a autonomia, impor a passividade, sujeitá-lo a baixas rendas, a desigualdade social ou no meio familiar, todos esses fatores são contribuintes para a privação dos idosos de assumirem uma vida social ativa, sendo isto responsável por agravos a sua saúde. Tais imposições estabelecidas quer seja pela sociedade ou pela família torna o idoso vulnerável à várias patologias, tanto de ordem biológica, quanto psicológica e social. (CANIZARES \& FILHO, 2011, P. 431), a aposentadoria tem se tornado um fator de risco para a saúde do idoso, pelo fato de ele agora ter que se adaptar à uma nova realidade social, por sofrer um processo gradativo de perdas, tanto pela falta do convívio com os colegas de trabalho, pelas responsabilidades agora não assumidas, e até mesmo agora pelos proventos a serem recebidos, o que pode the causar alterações psicológicas, desequilíbrio socioeconômico, podendo Ihe trazer algum agravo à saúde futuramente.

Conforme citado por (BARBOSA, 2012, P. 49), O fenômeno natural do envelhecimento dos idosos do Brasil tem se mostrado em uma triste realidade "de perdas de ordem afetiva, física e financeira", causando Ihes significativa 
desvalorização da sua importância como ser humano, como indivíduo na sociedade, sobre as suas ocupações, no seu dia a dia. Isto é compreensível quando visto de um prisma em que agora esse idoso vai depender de uma aposentadoria quase sempre não irá suprir as suas necessidades quando ainda tem que ajudar nas despesas quando morando com familiares, já não há alguém disponível para ouvir as suas queixas, sejam elas de que ordem for, pois na maioria dos casos os adultos vão trabalhar, e também já não tem o vigor do jovem ou do adulto, para andar, para sair do sedentarismo cotidiano. Muitas são as causas que podem deixar a população idosa em condições de vulnerabilidades às doenças e às suas complicações, como também à evolução para a morte, desde o seu envelhecimento natural, onde gradativamente $o$ organismo vai envelhecendo e com isto chegando às deficiências do funcionamento de cada órgão de cada sistema, e em consequência disto a fragilidade física, as deficiências respiratórias, vasculares, regenerativas, auditivas, visuais, olfativas, gustativas, sensitivas, digestivas, e também cognitivas, o fator ambiental como as condições de moradia, condições sanitárias, violência, outro fator importante é o socioeconômico, onde se incluem rendas, dívidas, escolaridade (GOMES ET ALL, 2013, P. 7), sedentarismo, também traz a sua parcela de contribuição neste processo, causando desgastes na esfera psicológica, gerando estresse. É muito comum encontrar alguém dizendo por aí que uma determinada pessoa vive doente, não cresceu, tem dificuldades em aprender, em entender determinados assuntos, em interagir socialmente, de falar em público, em expor suas opiniões, ser sempre passiva, aceitando tudo o que lhe impõe, porque passou muitas necessidades na vida, porque sofreu muito preconceito, porque foi muito discriminado. Isto nos leva a entender. que o indivíduo ao longo de sua vida, passou por tantos reveses que, se tornou vulnerável à diversos males, á várias doenças, tanto de ordem biológica, quanto também de ordem psicológica pelo desgaste mental, chamado de estresse. (LINDENMEYER, 2012, P. 344), "Victor Von Weizsacker defende a ideia que a história de cada sujeito é a base de todas as patologias orgânicas, promovendo desta forma uma reflexão que fomenta a união entre os aspectos subjetivos do sujeito e suas doenças". Para (BUENO \& SILVA, 2012, P. 2), há o entendimento de que o ser humano, diferente dos demais animais, 
existe e vive de uma integralidade de corpo e mente, o que é sensato atualmente considerar que não só os fatores biológicos são os responsáveis pelas causas de doenças físicas no corpo humano. As autoras ressaltam ainda que pela análise de seu estudo, houve a revelação de que doenças dermatológicas diversas ou psico dermatose, gastrintestinais, respiratórias, neoplásicas como o câncer e outras, são consideradas também resultado de fatores psicológico, chamadas de doenças psicossomáticas. Segundo as distintas autoras, existem explicações diversas sobre a influência do psicológico em causar e em desenvolver estas doenças; Como exemplo elas citam que as doenças da pele tem como fator desencadeante:"Depressão, stress, tensão emocional, dificuldade de simbolização, e de se expressar pela palavra", para as doenças gastrointestinais elas consideraram a não exteriorização emocional, o guardar as coisas dentro de si como sendo os agravantes, e para as doenças neoplásicas elas apontam como um das causas a "pobreza simbólica". (FACURY, 2011, p. 9, 13-14), cita que expressões comumente conhecidas como "nervos à flor da pele, vermelho de vergonha, pálido de susto, etc.", querem traduzir estados psíquicos, e que há décadas, há o reconhecimento científico pautado pelos relatos de pacientes, da importante contribuição tanto para o surgimento quanto para os agravos das doenças dermatológicas. Tais patologias resultantes desta interação entre a mente e a pele a autora chama de "distúrbios psico dermatológicos". A mesma cita que os distúrbios mais comuns envolvendo este maior órgão do corpo humano são os que afetam o couro cabeludo: alopécia, decorrente de um intenso quadro de estresse, tricotilomania, decorrente de tique nervoso, mais comum em crianças; O pigmento cutâneo: a melanina responsável em pigmentar a pele, é diminuída, causando o vitiligo, tendo como um dos causadores e ou agravantes o estresse e da depressão; As unhas: são as regiões mais frequentemente agredidas, onde o paciente roe-as na intenção de aliviar-se de uma tensão, podendo evoluir para uma compulsão; Peles sensíveis: Pode haver processo alérgico em decorrência de estado de tensão vivido pelo paciente, dermatite atópica, dermatite artefata ou automutilação, psoríase, etc. 


\section{SENESCÊNCIA E SENILIDADE}

O processo natural de envelhecimento é chamado de Senescência, pois é um processo que ocorre naturalmente, como já citados anteriormente, porém uma vez que este organismo se desgasta com o passar do tempo, o mesmo já não terá a mesma vitalidade que antes para exercer as suas funções, quando atingir uma idade mais avançada, tornando-o vulnerável a vários agravos à saúde, significando que o indivíduo terá predisposição para adoecer e isto será determinado pelo estilo de vida que cada um tem levado com passar do tempo. Uma vez instalado algum ou vários desses agravos à saúde, quer sejam de ordem psicológica, biológica, ou mesmo social, tem-se o processo da Senilidade. Este processo é caracterizado por manifestações problemas de ordem mental como depressão, demências, Alzheimer, entre outros, e de ordem corporal, como diversas doenças já citadas anteriormente, em todos os órgãos e sistemas. (CLOSAK ET ALL, 2011, P. 1765), Cita que mesmo havendo perdas nas esferas biológicas, social ou econômicas, não se justifica a afirmativa da ideia de que a velhice é dominada pela doença, pois para os autores manter o idoso em suas atividades e engajá-lo socialmente e no seio da família, lhe proporciona um envelhecimento saudável. Descartando a ideia de que todo idoso é senil. Por outro lado, a precocidade senil decorre de determinados fatores como: estilo de vida que desfavorecem à manutenção da saúde, baixa escolaridade, exclusão social e privação de suas atividades no ambiente familiar, e aposentaria, gerando conflitos emocionais e ou mesmo distúrbios corporais, como visto anteriormente.

\section{CONCLUSÃO}

Com o significativo aumento da longevidade da nossa população, surge a necessidade de capacitação profissional e de maior empenho e ou criação de políticas públicas de saúde voltadas para a assistência das suas necessidades, tanto psicológicas quanto orgânicas, e do conhecimento da problemática nas suas bases, as quais pelas vulnerabilidades podem trazer vários agravos à saúde desta população. Faz-se necessário o processo de educação continuada em geriatria e

Disponível em: https://www.nucleodoconhecimento.com.br/saude/doencaspsicossomaticas 
gerontologia, nos setores de saúde tanto público quanto privado, para o melhor entendimento dos fatores relacionados entre o corpo e a mente, causadores das doenças psicossomáticas, as quais podem levar o indivíduo ao processo de Senilidade precoce.

\section{REFERÊNCIAS BIBLIOGRÁFICAS}

ALONSO, F. R. B. ; Envelhecimento e vulnerabilidade: a inserção do idoso na família e o sentido dos fluxo inteegeracionais na geração de capital social. Universidade estadual de Campinas, fev. 2011. p. 1-219. Disponível em: http://www.bibliotecadigital.unicamp.br/document/? code=000793569. Acesso em 21/12/2015. BARBOSA, M. N. M.; Análise neuropsicológica para auxílio no diagnóstico diferencial entre a demência vascular subcortical, a doença de Alzheimer em estágio inicial e a depressão. Universidade de Brasília, instituto de Psicologia, 2012, p. 1-64. Disponível em: http://repositorio.unb.br/handle/10482/11237. Acesso em 20/12/2015 BUENO, L. M.; SIVA, L. C. O "psicologico" na causa e no desenvolvimento das doenças do corpo: o que dizem os artigos científicos produzidos no Brasil na última década. In: Anais v CIPSI - Congresso Internacional de Psicologia, Universidade estadual de Maringá/Pr. p. 1-2, 2012. Disponível em : http://eventos.uem.br/index.php/cipsi/2012/paper/viewFile/774/346. Acesso em 26/12/2015. CANIZARES, J. C. L.; FILHO, W. J. Fatores de risco à senilidade na transição à aposentadoria. Rev. bras. geriatr. Gerontol. Rio de Janeiro, v. 14, n. 3, p. 425-432, 2011 . Disponível em . acesso 04 Jan. 2016. CLOSAK, S. I.; BRAZ, E.; COSTA, M. F. B. N. A.; NAKANO, N. G. R.; RODRIGUES, J.; ALENCAR, R. A.; ROCHA, A. C. A. L. Senescência e senilidade: novo paradigma na Atenção Básica de Saúde. Rev. Esc. Enferm. USP, São Paulo, 2011, N. 45 (Esp. 2) p. 1763-8. Disponível em: http://dx.doi.org/10.1590/S0080-62342011000800022. Acesso em: 11/11/2015. DOLL, J.; RAMOS, A. C.; BUAES, C. S. Apresentação: educação e envelhecimento. In: educação \& realidade, Porto Alegre, v. 40, n. 1, p. 9-15, jan./mar. 2015. Disponível em: . Acesso em 20 dez. 2015. FACURY, T. C. C.; A escuta da psicanálise sobre a pele: uma abordagem da doença psicossomática. 
UFMG, Belo Horizonte, P. 9-118, 2011. Disponível em: http://www.bibliotecadigital.ufmg.br/dspace/bitstream/handle/1843/VCSA8HWFSQ/tereza_disserta_o_revisao_abril.pdf?sequence=1. Acesso em: 20/11/2015. GOMES, J. O.; ISHIKAWA, D. M.; HIRAI, M. R.; FLORA, R.; CRUZ, E. C.; GAGLIARDI, A. M. Z. A escolaridade pode influenciar na queixa subjetiva de memória em pacientes com ou sem demência?. VIII Congresso Sul Brasileiro de Geriatria e Gerontologia, jun. 2013, p. 2-120. Disponível em: http://sbgg.org.br/wpcontent/uploads/2014/10/GG_Anais2013.pdf acesso em 04/01/2016. GONÇALVES, C. D. Envelhecimento bem-sucedido, envelhecimento produtivo e envelhecimento ativo: Reflexões. Estudo interdisciplinar sobre o envelhecimento. Revista Envelhecimento vol. 20, n. 2, agosto 2015. pp. 645-57. Disponível em: http://www.seer.ufrgs.br/RevEnvelhecer/article/view/49428. Acesso em 24/10/2015. LINDENMEYER, C. Qual é o estatuto do corpo na psicanálise? tempo psicanalítico, Rio de Janeiro, v. 44.2, p. 341-359, 2012. Disponível em: http://pepsic.bvsalud.org/scielo.php?script=sci_arttextmd=S010148382012000200006. Acesso em 22/11/2015. SANTOS, G. S. A relação mente e corpo e a história da psicossomática. UNIFSJ, Itaperuna/RJ., 2011, P. 1-15. Disponível em: http://www.fsj.edu.br/wpcontent/uploads/2013/11/Arela\%C3\%A7\%C3\%A3o-corpo-e-mente-e-a-hist \%C3\%B3ria-da-Psicossomatica.pdf. Acesso em: 12/12/2015. SCHUMACHER, A. A.; PUTTINI, R. F.; NOJIMOTO, T. Vulnerabilidade, reconhecimento e saúde da pessoa idosa: autonomia intersubjetiva e justiça social. Saúde em debate. Rio de Janeiro, v. 3, n. 9, p. 281-93, abr./jun. 2013. Disponível em: http://www.scielo.br/pdf/sdeb/v37n97/v37n97a10.pdf. Acesso em 20/12/2015. 